Professor Dr. Frank Meyer, LL.M. (Yale), Zürich, und Professor Dr. Wolfgang Wohlers, Basel*

\title{
Tatprovokation quo vadis - zur Verbindlichkeit der Rechtsprechung des EGMR (auch) für das deutsche Strafprozessrecht
}

\author{
Der EGMR hat der auf eine Strafmilderung als Kompensation \\ für eine unzulässige Tatprovokation setzenden deutschen \\ Rechtsprechungspraxis die Konventionskonformität \\ abgesprochen. Die Verfasser legen dar, inwieweit die Vorgaben \\ des EGMR, nach denen ein Verwertungsverbot zwingend ist, \\ für die deutsche Strafjustiz verbindlich sind, und dass der \\ Versuch der 2. Kammer des Zweiten Senats des BVerfG, an \\ der bisherigen Linie festzuhalten, vor diesem Hintergund \\ nicht zu überzeugen vermag.
}

\section{Die Tatprovokation: Zankapfel zwischen Karlsruhe und Straßburg}

Bei der Bekämpfung des illegalen Handels mit Drogen, Waffen oder Menschen, der Prostitution, Korruption, Geldwäsche und der organisierten Kriminalität allgemein sind geheime Ermittlungsmaßnahmen, wie Telefonüberwachungen und verdeckte Ermittlungen, keine Ausnahmeerscheinungen, sondern Standardvorgehensweisen. ${ }^{1}$ Von Seiten der Strafrechtswissenschaft ist gerade der Einsatz verdeckter Ermittler und sonstiger V-Personen stets kritisch gesehen worden. ${ }^{2}$ Ihr Einsatz sei gekennzeichnet „durch eine heimliche, auf struktureller Täuschung basierende Form der Informationsgewinnung “3, mit der tief in die Privatsphäre der Zielpersonen und Dritter eingedrungen werde ${ }^{4}$. Auch unser akademischer Lehrer, Gerhard Fezer, dem wir diesen, unseren ersten gemeinsamen Aufsatz, in Dankbarkeit und Verehrung zu seinem ersten Todestag widmen, ${ }^{5}$ hat sich mit dieser Thematik befasst und eine tragfähige Begründung der verfassungsrechtlichen Zulässigkeit solcher heimlicher Ermittlungsmethoden eingefordert. ${ }^{6}$ Die Kritik, die am Einsatz ver-

\footnotetext{
* Frank Meyer ist Professor für Strafrecht und Strafprozessrecht unter Einschluss des internationalen Strafrechts an der Universität Zürich; Wolfgang Wohlers ist Professor für Strafrecht und Strafprozessrecht an der Juristischen Fakultät der Universität Basel.

** Zugleich Besprechung von EGMR v. 23.10.2014 - Furcht v. Deutschland - 54648/09 (abrufbar unter: http://wwwe.echr.coe.int); sowie BVerfG v. 18.12.2014 - 2 BvR 209/14, 240/14 und 262/14 (abrufbar unter: bttp:// wrwe.bundesverfassungsgericht.de).

1 Hauck, Heimliche Strafverfolgung und Schutz der Privatheit, 2014, S. 51 f.; Zöller StraFo 2008, 15, 16.

2 Vgl. hierzu grundlegend Lüderssen, in: Festschrift für Peters, 1974, S. 349 ff.; sowie jüngst die monografische Arbeit von Tyszkiewicz, Tatprovokation als Ermittlungsmaßnahme. Rechtliche Grenzen der Beweiserhebung und Beweisverwertung beim Einsatz polizeilicher Lockspitzel im Strafverfahren, 2014, passim.

3 Frister, Handbuch des Polizeirechts, 5. Aufl. 2012, Kap. F Rn. 316.

4 Fezer JZ 1995, 972; eingehend Hauck (Fn. 1).

5 Wolfgang Wohlers war von 1988 bis 1992 am Lehrstuhl Fezer als wissenschaftlicher Mitarbeiter tätig, Frank Meyer von 2000 bis 2003. Das materiellrechtliche Habilitationsprojekt von Wolfgang Wohlers wurde von Kurt Seelmann (Universität Hamburg und Universität Basel) und das Habilitationsprojekt von Frank Meyer von Martin Böse (Universität Bonn) betreut. Unsere rechtswissenschaftliche Grundprägung haben wir aber beide durch Gerhard Fezer erfahren. Die Zeit am Lehrstuhl Fezer war für uns beide eine persönlich und fachlich prägende Erfahrung, die in ihrer Bedeutung nicht überschätzt werden kann (vgl. auch die Nachrufe auf Gerhard Fezer in JZ 2014, 947 [Woblers] und in HRRS 2014, 394 [Meyer]). 6 Fezer JZ 1995, 972.
}

deckter Ermittler und insbesondere an der Provokation von Taten geäußert worden ist, hatte aber ganz offenbar nie eine Chance, sich gegen die Bedürfnisse der Strafverfolgungspraxis durchzusetzen, die geradezu mantraartig die kriminalistische Notwendigkeit dieser Ermittlungsinstrumente betont. $^{7}$

Im Zuge dieser Entwicklung haben sich Ermittlungsmethoden wie die Tatprovokation etabliert, die als typische Ermittlungstaktiken verdeckter Ermittler und sonstiger VPersonen angesehen werden. ${ }^{8}$ Ein Provokations- oder Verleitungsmoment (bezüglich neuer Straftaten) ist indes mit verdeckten Ermittlungen nicht zwingend verbunden. Charakteristikum heimlicher Ermittlungsmaßnahmen ist die gezielte Ausnutzung der Unkenntnis des Informationsträgers über die laufende Ermittlung ${ }^{9}$ zur Gewinnung von Informationen über eine möglicherweise begangene Tat. Bei der Tatprovokation geht es aber nicht um die Infiltration einer Verbrechensstruktur zur Informationsgewinnung, sondern um die Stimulierung und Auslösung einer Tat. Dabei kann die ermittlungstaktische Bedeutung einer Tatprovokation enorm variieren. ${ }^{10}$ Sie kann zum Zwecke der Verurteilung wegen der provozierten Tat oder zum Zwecke der Ermöglichung einer Verurteilung wegen zurückliegender Taten durch Überführung von Serientätern erfolgen; sie kann auf die Absicherung von Ermittlungen durch Erlangung von Beweisen hinsichtlich des modus operandi oder zu Hintermännern und Drahtziehern abzielen, und sie kann auch als Türöffner für die Kronzeugenregelung oder zur Verhütung geplanter Taten dienen. Zielt der Einsatz des Lockspitzels auf unverdächtigte Personen ab oder soll er der Prüfung des Normgehorsams einer möglicherweise tatgeneigten Person dienen, liegen die Voraussetzungen für ein staatliches Tätigwerden auf der Grundlage der StPO von vornherein nicht vor. ${ }^{11}$ Obwohl die Tatprovokation damit prima vista kein per se strafprozess- und verfassungsrechtlich akzeptables Instrument sein kann, haben das $B \operatorname{Verf} G^{12}$ und der $B G H^{13}$ stets betont, dass insbesondere die Bekämpfung des Drogenhandels ohne den Einsatz verdeckter Ermittlungsmethoden

$7 \quad B \operatorname{VerfG}$ NJW 1985, 1767; NJW 1992, 168; BGHSt 32, 115 = JZ 1984, 430 mit Anm. Fezer; 47, 44, 1; Möbrenschlager wistra 1992, 326, 330; Koriath Kriminalistik 1996, 535, 536

8 Frister (Fn. 3), Kap. F Rn. 336; Maul, in: Festschrift BGH, 2000, S. 569; zum Erkenntnisstand betreffend den Einsatz polizeilicher Vertrauenspersonen vgl. Kreuzer, in: Festschrift für Schreiber, 2003, S. 228 ff. m. w. N.; zum praktischen Nutzen Lüderssen, Festgabe BGH, Bd. IV, 2000, S. 883, 887; Tyszkiewicz (Fn. 2), S. $92 \mathrm{ff}$.

$9 \operatorname{Hauck}($ Fn. 1), S. 697.

10 von Danwitz, Staatliche Straftatbeteiligung, 2005, S. 38 f.; Lüderssen, in: Festschrift für Peters, 1974, S. 349, 350 ff.; Maul/Fischer NStZ 1992, 7 , 10; Rieble KrimJ 1985, 44, $48 \mathrm{f}$.

11 Vgl. auch von Danwitz (Fn. 10), S. 93; Maul/Fischer NStZ 1992, 7, 10 f.; Tyszkiewicz (Fn. 2), S. 234.

12 Vgl. $B \operatorname{VerfGE} 57,250,284 ; B \operatorname{VerfG}$ NJW 1987, 1874, 1875; 1995, 651, 652.

13 BGHSt 32, 115, 121 f.; 32, 345, 346 = JZ 1986, 98 (dazu Schumann JZ 1986, 66; Anm. Meyer NStZ 1984, 134); BGHSt 41, 42, 43 = JZ 1995, 970 mit Anm. Fezer; 45, 321, 324; 47, 44, 50; BGH StV 1995, 354, 365; BGH NJW 1981, 1626 m.w.N. 
nicht auskommen kann, ${ }^{14}$ und vor diesem Hintergrund auch die staatliche Tatprovokation als ein Vorgehen eingestuft, das nicht grundsätzlich zu verwerfen ist, sondern bei dem nur gewisse Grenzen einzuhalten sind. Aber auch dann, wenn diese Grenzen überschritten werden, soll dies grundsätzlich weder ein Verfahrenshindernis begründen, noch unterliegen die gewonnenen Erkenntnisse einem Verwertungsverbot; die unzulässige Tatprovokation hat vielmehr nur eine Strafmilderung zur Folge. Ein Verfahrenshindernis könne nur in Extremfällen angenommen werden, weil das Rechtsstaatsprinzip auch das Interesse an einer der materiellen Gerechtigkeit dienenden Strafverfolgung schütze. ${ }^{15}$ Dieses Lösungsmodell weist aus der Sicht der Strafverfolgung zwei große Vorteile auf: Erstens ist die Tatprovokation grundsätzlich zulässig und eröffnet damit Ermittlungsansätze; und zweitens haben die Gerichte maximale Freiheit bei der Frage, wie auf etwaige Grenzüberschreitungen der Ermittlungsorgane zu reagieren ist: Sie können schon dadurch steuern, dass die Tatprovokation als noch zulässig oder bereits unzulässig eingestuft wird, und sie haben auch dann, wenn die Tatprovokation die Grenzen des noch Zulässigen überschritten hat, die Möglichkeit, die Konsequenzen im Rahmen zu halten.

Vor diesem Hintergrund muss die Entscheidung des $E G M R$ in der Sache Furcht gegen Deutschland als Fanal eingestuft werden. ${ }^{16}$ Der Gerichtshof vertritt die Auffassung, dass die Strafzumessungslösung nicht mit Art. 6 EMRK vereinbar ist, ${ }^{17}$ angezeigt sei vielmehr ein strenges Verwertungsverbot. ${ }^{18}$ Selbst eine substantielle Strafmilderung könne den Verstoß gegen den Fair-trial-Grundsatz nicht hinreichend kompensieren. Der EGMR diktiert den Vertragsstaaten grundsätzlich nicht die Art und Weise der Umsetzung seiner Urteile, sondern eröffnet ihnen die Möglichkeit einer struktur- und prinzipienadäquaten Einpassung in die eigene Verfahrensordnung, soweit der materielle Kern der Vorgaben effektiv verwirklicht wird. Bezüglich der funktionalen Äquivalenz herrschen jedoch offensichtlich ganz unterschiedliche Auffassungen. Zugrunde liegt ihnen eine stark abweichende Einschätzung der Schwere des Verstoßes. Vorliegend geht der EGMR den ungewöhnlichen Schritt, die Eignung eines bestimmten Kompensationsmittels von vornherein auszuschließen, um damit den Spielraum der Mitgliedstaaten entscheidend zu begrenzen. Der EGMR lässt zwar im Sinne einer salvatorischen Klausel alternativ auch ähnlich wirkende Verfahrensweisen zu, doch müssen sich diese an der Wirkungsweise eines strengen Beweisverwertungsverbots messen lassen. Die Entscheidungsgründe legen es nahe, dass nun alles davon abhängt, ob sich die Strafverfolgungsorgane im Rahmen des Zulässigen gehalten haben. Haben sie die Grenzen überschritten, muss ein Verwertungsverbot für die in Frage stehenden Erkenntnisse angenommen werden, was vor dem Hintergrund der Praxis der Strafverfolgung die Frage aufwirft, ob dieses Verwertungsverbot nicht de facto

14 Kritisch zur Orientierung an Zweckmäßigkeitsgesichtspunkten bereits Wolter, in: Festgabe BGH, Bd. IV, 2000, S. 982 f.; zustimmend dagegen Krey, in: Festschrift für Kohlmann, 2003, S. 651.

$15 B \operatorname{VerfG}$, Beschluss v. 18.12.2014 - 2 BvR 209/14, 240/14, 262/14, Rz. 38.

$16 E G M R$ v. 23. 10.2014 - Furcht v. Deutschland - 54648/09. Die Erwartung, dass letztlich der EGMR die auf die Strafzumessungslösung festgelegte Rechtsprechung aufbrechen werde, hat Kreuzer bereits im Jahre 2003 geäußert; vgl. Kreuzer, in: Festschrift für Schreiber, 2003, S. 228, 242 f.; Paeffgen, in: SK-StPO, 5. Aufl. 2015, Anh. \$206 Rn. 25). Skeptisch zur Möglichkeit, die deutsche Rechtsprechung über den EGMR zu beeindrucken, Lüderssen, in: Festgabe BGH, Bd. IV, 2000, S. 886.

$17 E G M R$ v. 23. 10. 2014 - Furcht v. Deutschland - 54 648/09, Rn. 64, 69. $18 E G M R$ v. 23. 10. 2014 - Furcht v. Deutschland - 54648/09, Rn. 64. ein Verfahrenshindernis begründet - weil keine verwertbaren Beweise mehr vorhanden sind, die eine Fortsetzung des Verfahrens erlauben würden. Und genau diese strikte Rechtsfolge dürfte der Grund dafür sein, dass das BVerfG nur rund zwei Monate nach der Entscheidung des EGMR in einem Beschluss vom 18.12.2014 nochmals betont hat, dass eine rechtsstaatswidrige Tatprovokation auch unter Berücksichtigung der neuen Rechtsprechung des EGMR einer Verurteilung wegen der provozierten Taten nicht grundsätzlich entgegensteht. ${ }^{19}$

Das Verhältnis der nationalen (Höchst-)Gerichte zum $E G M R$ ist in den letzten Jahren in verschiedenen Mitgliedstaaten konfliktbehaftet. Nachdem es den Anschein hatte, dass das $B V \operatorname{erf} G$ durch die Präzisierung des verfassungsrechtlichen Umsetzungskorridors und seiner methodischen Herangehensweise für spürbare Entspannung gesorgt hat, ${ }^{20}$ könnte sich nun am Umgang mit der Tatprovokation neuerlicher Streit entzünden, wobei es nicht nur um die „richtige“ Rechtsfolge für einen Fair-trial-Verstoß geht. Der Meinungsverschiedenheit liegen deutlich unterschiedliche Vorstellungen davon zugrunde, wie schwer der in Frage stehende Eingriff wiegt und unter welchen Voraussetzungen der Einsatz einer Tatprovokation überhaupt legitim sein kann. Aber auch rechtsmethodisch lässt die Argumentation des BVerfG aufhorchen, deutet sie doch einen Weg an, der sich bei Bedarf aktivieren ließe, um eine unliebsame EGMR-Rechtsprechung zu konterkarieren. Die Gerichtshöfe anderer Europaratsstaaten werden diese Ausführungen aufmerksam zur Kenntnis nehmen.

\section{Die unzulässige Tatprovokation aus Sicht der bundesdeutschen Strafjustiz}

Die Bedeutung, die der Tatprovokation für die Praxis zukommt, wird durch zwei Aspekte determiniert: es geht darum, wie weit verdeckt ermittelnde Personen bei der Einwirkung auf die Zielperson gehen dürfen, und was die Konsequenzen sind, wenn diese Grenze überschritten wird. Die deutschen Strafgerichte haben die Schwelle des Unzulässigen stets im Vagen gehalten, sie jedenfalls aber eher hoch angesetzt und selbst bei einer Überschreitung dieser Schwelle allenfalls eine Strafmilderung in Betracht gezogen.

\section{Die Unzulässigkeit einer Tatprovokation aus Sicht der deutschen Rechtsprechung}

Die in der Literatur geforderte Anerkennung der grundsätzlichen Unzulässigkeit des Lockspitzeleinsatzes ${ }^{21}$ war für die Rechtsprechung nie eine ernsthafte Option. Es ging stets allein darum, dem als notwendig angesehenen Lockspitzeleinsatz Grenzen zu setzen, wobei die Rechtsprechung als Grundlage auf das Rechtsstaatsprinzip rekurriert, das es den Strafverfolgungsorganen untersage, „auf die Verübung von Straftaten hinzuwirken, wenn die Gründe dafür vor diesem Prinzip nicht bestehen können“.22 Der $B G H$ hat diesbezüglich zunächst auf eine Gesamtschau abgestellt, in die jedenfalls die Grundlage und das Ausmaß des Tatverdachts, die Art und

$19 B \operatorname{VerfG}$, Beschluss v. 18. 12.2014 - 2 BvR 209/14, 240/14, 262/14. 20 Positive Reaktionen in EGMR v. 9.6.2011 - Schmitz v. Deutschland $30493 / 04$, Rn. 41; EGMR v. 19.1.2012 - Kronfeldner v. Deutschland 21 906/09, Rn. 59.

21 Vgl. zuletzt Tyszkiewicz (Fn.2), S. $128 \mathrm{ff}$. für die Provokation von Straftaten zum Zwecke der Aburteilung dieser Taten.

$22 B G H$ NJW 1980, 1761; vgl. auch BGHSt 32, 345, 346; BGH NStZ 1981, 70; $B G H$ NJW 1981, 1626 mit krit. Anm. Mache StV 1981, $599 \mathrm{ff}$; $B G H \mathrm{StV}$ 1995, 364, 365. 
Intensität der Einflussnahme durch den Lockspitzel sowie die Tatbereitschaft der Zielperson sowie die von dieser entfalteten Aktivitäten einzubeziehen waren. ${ }^{23}$ Unzulässig sollte die Einwirkung auf die Zielperson erst dann sein, wenn das Verhalten des Lockspitzels ein solches Gewicht erlangt, dass der eigene Beitrag der Zielperson demgegenüber in den Hintergrund tritt, ${ }^{24}$ was zunächst allenfalls für Fälle angenommen wurde, in denen die Zielperson so unter Druck gesetzt worden war, dass man übersetzt in die Kategorien der Beteiligungslehre davon reden kann, dass der Lockspitzel die Zielperson als sein Werkzeug benutzt oder mit dieser quasi-mittäterschaftlich zusammengewirkt hatte. ${ }^{25}$ So sah sich der 2. Strafsenat des $B G H$ in einem der wenigen Fälle, in denen eine unzulässige Tatprovokation angenommen wurde, veranlasst, darauf hinzuweisen, dass das Verhalten eines Lockspitzels

„die Grenze des Zulässigen nicht nur dann [überschreitet], wenn er einen anderen so in seiner Gewalt hat, dass dieser unbedingt seinen Wünschen nachkommen muss, was die Strafkammer offenbar voraussetzt. Auch wenn er den anderen mit der hier festgestellten Intensität zum (erneuten) strafbaren Tun veranlasst sowie im weiteren Verlauf die Tat in jeder Phase mit beherrscht und steuert, erscheint der den staatlichen Organen zuzurechnende Tatbeitrag - auch bei Berücksichtigung der vom Angeklagten später entwickelten Aktivitäten - unvertretbar übergewichtig." 26

Im Jahre 1999 modifizierte der $B G H$ seine Rechtsprechung - in Reaktion auf die Entscheidung des EGMR in der Sache Teixeira ${ }^{27}$ - dahingehend, dass sich die Unzulässigkeit der Tatprovokation maßgebend aus dem Umstand ableiten ließ, dass im Zeitpunkt der Einwirkung durch den Lockspitzel keine Anhaltspunkte dafür vorlagen, dass die Zielperson ,in einem den $\int \mathbb{S} 152$ Abs. 2, 160 StPO vergleichbaren Grad verdächtig ist, an einer bereits begangenen Straftat beteiligt gewesen zu sein oder zu einer zukünftigen Straftat bereit zu sein". ${ }^{28}$ Somit soll neben dem auf eine bereits begangene Straftat bezogenen strafprozessualen Anfangsverdacht auch der gänzlich anders gelagerte, prospektiv an deliktsspezifischen Gegebenheiten ${ }^{29}$ ausgerichtete Verdacht genügen, dass die Zielperson geneigt sein könnte, eine entsprechende Straftat zu begehen. ${ }^{30}$

Eine unzulässige Tatprovokation kann nach alledem praktisch nur in drei Fällen vorliegen: ${ }^{31}$ Erstens in den Fällen, in denen Lockspitzel „auf gut Glück“ gegen sowohl strafprozessual unverdächtige als auch polizeirechtlich gesehen nicht als tatgeneigt einzustufende Personen vorgehen ${ }^{32}$ - wobei allerdings auch in diesen Fällen das bloße Ansprechen, ob

$23 B G H$ NJW 1980, 1761; 1981, 1626; BGH NStZ 1981, 70; 1984, 78, 79; vgl. auch Bruns NStZ 1983, 49, 54 ff.; Kinzig StV 1999, 288, 290; K. Meyer NStZ 1984, 134; kritisch zur Vagheit des Kriterienkatalogs Seelmann ZStW 95 (1983), 797, 833.

24 BGHSt 32, 345, 347 mit Bespr. Bruns StV 1984, $388 \mathrm{ff}$;; BGH NJW 1981, 1626; BGH NStZ 1982, 126.

25 Nach Tyszkiewicz (Fn. 2), S. 123, wurden allenfalls „besonders krasse Fälle“ erfasst; die Rechtsprechung habe dem Lockspitzeleinsatz kaum noch Grenzen gesetzt.

26 BGH NStZ 1984, 78, 79; vgl. auch den Fall, der den Entscheidungen BGHSt 32, 345 sowie LG Stuttgart StV 1984, 197 zugrunde lag und bei dem es auch um die Provokation einer vollständig unbescholtenen Person ging.

27 So auch Tyszkiewicz (Fn. 2), S. 125.

28 BGHSt 45, 321, $337=\mathrm{JZ} 2000,363$ mit Anm. C. Roxin; vgl. auch $B G H$ $\mathrm{StV}$ 1995, 364, 365 sowie van Gemmeren, in: NJW Sonderheft für G. Schäfer, 2009, S. 30; Maul, in: Festschrift BGH, 2000, S. 569, 575; Bruns, in: Karlsruher Kommentar (KK) StPO, 7. Aufl. 2013, \$110c Rn. 10.

29 Vgl. BGHSt 47, 44, 51.

30 Tyszkiewicz (Fn. 2), S. 126.

31 Vgl. auch Wolter, in: Systematischer Kommentar (SK) zur StPO, 4. Aufl. 2010, $\mathbb{S} 110$ c Rn. 9a; sowie I. Roxin, in: Festschrift für Beulke, 2015, S. 987,989 .

32 BGHSt 45, 321, 326 und 337f.; vgl. auch BGH StV 1995, 131; 1995, 364; Wolter, in: SK-StPO (Fn. 31), $\ 110$ c Rn. 9a; Gaede/Buermeyer HRRS 2008, 279, 280. die Zielperson Betäubungsmittel beschaffen/verkaufen könne, noch nicht als Tatprovokation gelten soll;33 zweitens in den Fällen einer auch gemessen am vorhandenen Verdacht absolut übergewichtigen Einflussnahme auf einen Verdächtigen, zum Beispiel durch Todesdrohungen; ${ }^{34}$ und drittens in den Fällen, in denen der Lockspitzel den vorhandenen Tatentschluss qualitativ deutlich intensiviert, das heißt die Zielperson in eine Tat verstrickt, die im Vergleich zu ihrer ursprünglichen Tatbereitschaft einen erheblich gesteigerten Unrechtsgehalt aufweist. Der insoweit erforderliche (von der bestehenden Verdachtslage nicht mehr getragene) "Quantensprung“ kann sich aus einem Übergang vom Handel mit weichen Drogen zu einem solchen mit harten Drogen ergeben oder aus der Steigerung der Menge, mit der gehandelt wird ${ }^{35}$ - wobei auch hier wieder die Fälle ausgeklammert werden, in denen die Zielperson auf das Ansinnen ohne weiteres eingeht. ${ }^{36}$

\section{Die Konsequenzen einer unzulässigen Tatprovokation aus Sicht der deutschen Rechtsprechung}

Die Praxis, Fälle unzulässiger Tatprovokation mit einer Strafmilderung zu kompensieren, ist im Schrifttum auf Kritik gestoßen, weil diese nichts daran ändern könne, dass das Verfahren insgesamt gesehen ab initio unfair ist und damit gegen Art. 6 EMRK und das Rechtsstaatprinzip verstoße. ${ }^{37}$ Nichtsdestotrotz hat die stattdessen vorgeschlagene Annahme eines schuldunabhängigen Strafausschließungsgrunds, ${ }^{38}$ eines Verfahrenshindernisses ${ }^{39}$ oder eines Verwertungsverbotes ${ }^{40}$ in der Rechtsprechung keinen Anklang gefunden.

33 Vgl. BGHSt 45, 321, 335, insoweit zust. C. Roxin JZ 2000, 369, 370; BGHSt 47, 44, 47; vgl. auch van Gemmeren, in: NJW Sonderheft für G. Schäfer, 2009, S. 29f.; I. Roxin, in: Festschrift für Beulke, 2015, S. 989; Bruns, in: KK-StPO (Fn. 28), \110c Rn. 10; Gercke, in: Heidelberger Kommentar (HK) StPO, 5. Aufl. 2012, $\$ 110$ c Rn. 9; Löffelmann, in: Anwaltskommentar (AnwK) StPO, 2. Aufl. 2010, $\$ 110$ c Rn. 9; restriktiver Fischer/ Maul NStZ 1993, 7, $10 \mathrm{ff}$.

34 BGH NStZ 2009, 405, 406; BGH StV 1995, 131; StV 1995, 364; StV 1981, 163, 164; vgl. auch BGHSt 47, 44, 48 f.

35 BGHSt 47, 44, 49 f.; vgl. auch Gaede/Biermeyer HRRS 2008, 279, 280; van Gemmeren, in: NJW Sonderheft für G. Schäfer, 2009, S. 30; Tyszkiewicz (Fn. 2), S. 126 f.; Bruns, in: KK-StPO (Fn. 28), \110c Rn. 10; Wolter, in: SKStPO (Fn. 31), $\mathbb{1} 110$ c Rn. 9a. Der Übergang von Kokain auf Heroin soll dabei wohl nicht genügen; vgl. $B G H$ v. 11.12.2013 - 5 StR $240 / 13=$ HRRS 2014 Nr. 163.

36 BGHSt 47, 44, 51.

37 Gaede/Buermeyer HRRS 2008, 279, 285; Krewzer, in: Festschrift für Schreiber, 2003, S. 228, 241 f.; F. Meyer, in: Karpenstein/Mayer (Hrsg.), EMRK, 2. Aufl. 2015, Art. 6 Rn. 157; Sinner/Kreuzer StV 2000, 114, 116 f.; Tyszkiewicz (Fn. 2), S. $216 \mathrm{f}$.

$38 \mathrm{Vgl}$. insbesondere Seelmann ZStW 95 (1983), 797, 831; C. Roxin JZ 2000, 369 f.; Wolter NStZ 1993, 1, 10; ders., in: Festgabe BGH IV, 2000, S. 980; Wolter, in: SK-StPO (Fn. 31), $\$ 110$ c Rn. 11; Beulke, in: Satzger/ Schluckebier/Widmaier (SSW), StGB, 2. Aufl. 2014, Einl. Rn. 110; kritisch hierzu Tyszkiewicz (Fn. 2), S. 212; ablehnend K. Meyer NStZ 1984, 134, 135; Schumann JZ 1986, 66, 71; vgl. aber auch Roxin/Schünemann, Strafverfahrensrecht, 28. Aufl. 2014, $\mathbb{S} 37$ Rn. 8 mit dem Vorschlag einer zwingenden Verfahrensverbindung und Bestrafung von Provozierendem und agent provocateur.

39 Bruns NStZ 1983, 49, 52 f.; ders. StV 1984, 388, 390 f.; Dencker, in: Festschrift für Dünnebier, 1982, S. 453; Endriß/Kinzig StraFo 1998, 299, 303; Eschelbach StV 2000, 390, 395; Esser, Auf dem Weg zu einem europäischen Strafverfahrensrecht, 2002, S. 176; Maul, in: Festschrift BGH, 2000, S. 569, 577 f.; Schumann JZ 1986, 66, 70 ff.; Sinner/Kreuzer StV 2000, 114, 117; Taschke StV 1984, 178 f.; Tyszkiewicz (Fn. 2), S. 223 ff.; a. A. Foth NJW 1984, $221 \mathrm{f} . ;$ K. Meyer NStZ 1984, 134; Radtke in: Radtke/Hohmann (Hrsg.), StPO, 2011, Einl. Rn. 63; Beulke, in: SSW-StGB (Fn. 38), Rn. 288; zur Begründung eines Verfahrenshindernisses bei extremen Verstößen gegen den Fair-trial-Grundsatz vgl. auch Weiler GA 1994, 561, $582 \mathrm{ff.}$

40 Grundlegend Lüderssen, in: Festschrift für Peters, 1974, S. 349, 366 ff.; ders. StV 2002, 169; vgl. auch Wolter, in: SK-StPO (Fn. 31), \110c Rn. 11; sowie Ambos NStZ 2002, 628, 632; Bruns StV 1984, 388, 392 f.; Eschelbach 
Das $B \operatorname{VerfG}$ hat die Möglichkeit eines Verfahrenshindernisses zwar für zunächst nicht näher definierte „Ausnahmefälle" nicht ausgeschlossen ${ }^{41}$ und will sich auch in seinem jüngsten Kammerbeschluss nicht festlegen. ${ }^{42}$ Es lässt aber durchblicken, wie ein solcher Extremfall aussehen müsste, um hypothetisch ein Verfahrenshindernis auslösen zu können. Ein gänzlich Unverdächtiger müsste provoziert worden sein und lediglich als Objekt der staatlichen Ermittlungsbehörden einen vorgefertigten Tatplan ohne eigenen Antrieb ausgeführt haben. Doch selbst für einen derart krassen staatlichen Exzess scheut die 2. Kammer des Zweiten Senats am Ende eine klare Aussage darüber, ob eine solche Situation zwingend als Extremfall zu behandeln wäre.

Die Strafgerichte der Bundesrepublik haben zwar zunächst noch kurz geschwankt, ${ }^{43}$ dann aber die Annahme eines Verfahrenshindernisses ausdrücklich als einen nicht sachgerechten „Alles oder Nichts“-Ansatz ${ }^{44}$ zurückgewiesen. ${ }^{45}$ Auch die Lösung über ein Verwertungsverbot wurde verworfen, wobei der $B G H$ vordergründig auf die Schwierigkeiten bei der Bestimmung der Reichweite des Verwertungsverbots verweist, ${ }^{46}$ letztlich aber auch hier der eigentliche Grund der sein dürfte, dass das Verwertungsverbot im Ergebnis zu einer Verfahrenseinstellung zwingen würde. ${ }^{47}$ Im Anschluss an BGHSt 32, 345 setzt die Rechtsprechung nun einheitlich auf eine Strafzumessungslösung: 48 Die unzulässige Tatprovokation begründet einen schuldunabhängigen Strafmilderungsgrund, der auch die Unterschreitung der sonst - also ohne die Tatprovokation - für die in Frage stehende Tat eigentlich schuldangemessenen Strafe ermöglichen soll, ${ }^{49}$ wobei das Ausmaß der Milderung ausdrücklich im Urteil festzuhalten sei. ${ }^{50}$ In der neueren Diskussion wird erwogen, dass auch für die Strafmilderung infolge einer un-

StV 2000, 390, 396; Fischer/Maul NStZ 1992, 7, 13; Franzheim NJW 1979, 2014, 2015; Kinzig StV 1999, 288, 292; und jüngst Tyszkiewicz (Fn.2), S. 224 f.; vgl. auch Sinner/Krenzer StV 2000, 114, 117; ablehnend hierzu $K$. Meyer NStZ 1984, 134 f.; Maul, in: Festschrift BGH, 2000, S. 569, 576 f.; Radtke, in: Radtke/Hobmann (Fn. 39), Einl. Rn. 63.

$41 B$ VerfG NJW 1995, 651, 652; vgl. auch bereits BVerfG NJW 1986, 1427; 1987, 1874 f.; BVerfG NStZ 1985, 131

$42 B \operatorname{VerfG}$, Beschluss v. 18.12.2014 - 2 BvR 209/14, 240/14, 262/14, Rz. 34, 38.

43 Vgl. $B G H$ NJW 1981, 1626 f.; sowie $B G H$ NStZ 1982, 126; vgl. auch die Nachweise bei Paeffgen, in: SK-StPO (Fn. 16), Anh. $\$ 206$ Rn. 25 Fn. 161; sowie Weiler GA 1994, 561, 565.

44 So ausdrücklich BGHSt 45, 321, 334; vgl. auch van Gemmeren, in: NJW Sonderheft für G. Schäfer, 2009, S. 29

45 Grundlegend hierzu die Entscheidungen BGHSt 32, 345, $350 \mathrm{ff}$.; sowie BGHSt 45, 321, 333 f.; vgl. aber auch schon BGHSt 33, 356, 362; dezidiert a. A. Maul, in: Festschrift BGH, 2000, S. 569, 578 f.; vgl. auch C. Roxin JZ $2000,369,370$.

46 Vgl. BGHSt 45, 321, 335; zutreffende Kritik an der Argumentation des $B G H$ bei Tyszkiewicz (Fn. 2), S. 222 f.; ablehnend zur Annahme eines Beweisverbotes auch schon BGHSt 32, 345, 355.

47 Dies betonen auch Kinzig StV 1999, 289, 290; Tyszkiewicz (Fn.2), S. 223 .

48 BGHSt 32, 345, 355f. mit krit. Bespr. Bruns StV 1984, 388, 392 und Schumann JZ 1986, 66, ff.; Meyer NStZ 1985, 134; vgl. auch BGHSt 45, 321, 324 ff. m. w. N. sowie die umfassenden Nachweise bei Paeffgen, in: SK-StPO (Fn. 16), Anh. $\$ 206$ Rn. 25 Fn. 162-165; vgl. auch Herzog StV 2003, 410, 411; Maul, in: Festschrift BGH, 2000, S. 569, 571; Roxin/Schünemann (Fn. 38), $₫ 37$ Rn. 7; Bruns, in: KK-StPO (Fn. 28), $\$ 10$ c Rn. 11.

49 BGHSt 45, 321, 339; BGH StV 1994, 368, 369; 1995, 131; 1995, 364, 365; kritisch zur Strafzumessungslösung Bruns MDR 1987, 177 ff.; Jahn, Gutachten zum 67. DJT, 2008, C 107; Kinzig StV 1999, 288, 290; Lüderssen, in: Festgabe BGH, Bd. IV, S. 889 f.; Sommer NStZ 1999, 48, 49 f.; Wolter, in: SK-StPO (Fn. 31), $\$ 110$ c Rn. 10.

50 BGHSt 45, 321, 335 f., 339; BGH NStZ 2013, 99, 100; Bruns, in: KK-

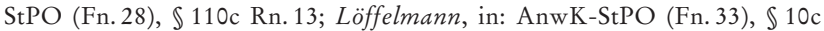
Rn. 10; Gercke, in: HK-StPO (Fn. 33), $\int 110$ c Rn. 9; van Gemmeren, in: NJW Sonderheft für G. Schäfer, 2009, S. 31; vgl. auch Sinner/Kreuzer StV 2000, 114, 115 mit einem Verweis darauf, dass dies zur Disziplinierung der Strafverfolgungsorgane notwendig sei. zulässigen Tatprovokation die vom $B G H$ ursprünglich für die Fälle der Verletzung des Beschleunigungsgrundsatzes kreierte Vollstreckungslösung zur Anwendung zu kommen hat. ${ }^{51}$ Diese Strafzumessungslösung ist aus Sicht des $B G H$ „geeignet, den im Einzelfall erforderlichen Ausgleich für den Konventionsverstoß zu schaffen“. 52

\section{Die unzulässige Tatprovokation aus der Sicht des EGMR}

Grundsätzlich abweichend vom Ansatzpunkt der deutschen Gerichte geht der EGMR davon aus, dass das öffentliche Interesse an einer wirksamen Verbrechensbekämpfung „cannot justify the use of evidence obtained as a result of police incitement, as to do so would expose the accused to the risk of being definitely deprived of a fair trial from the outset" ${ }^{\text {“ }} 53$ Konsequenterweise geht der EGMR dann auch davon aus, dass Beweismaterial, welches durch eine unzulässige Tatprovokation geschaffen wurde, nicht im Rahmen der Strafverfolgung dieser Tat verwendet werden darf. ${ }^{54}$ Der Gerichtshof konzediert zwar, dass grundsätzlich auch andere angemessene Mittel vorstellbar sind, um der Rechtsverletzung abzuhelfen. Jedoch reduziert er den Kreis möglicher Rechtsfolgen auf Verwertungsverbot, Strafausschließungsgrund (entrapment) oder ähnlich wirksame Verfahrensweisen. ${ }^{55}$ Eine Strafmilderungslösung kommt dagegen - wie der EGMR in der Rechtssache Furcht nun auch ausdrücklich festgehalten hat nicht in Betracht. 56

\section{Umfang des Beweisverwertungsverbots}

In der Spruchpraxis wird stets die Unverwertbarkeit der bemakelten Beweismittel betont. Nicht eindeutig hat sich der EGMR dagegen zum Umfang des Beweisverwertungsverbots erklärt. Dieser Umstand mag das $B \operatorname{Verf} G$ dazu verleitet haben, ein mögliches Verwertungsverbot allein auf die Aussagen der verdeckt handelnden Personen zu bezie-

51 Vgl. Kraatz JR 2008, 189, 194; Weber JR 2008, 36, 38; kritisch Gaede JZ 2008, 422, 424; Streng JZ 2008, 979, 986; Tyszkiewicz (Fn. 2), S. 221 f.; sowie jüngst I. Roxin, in: Festschrift für Beulke, 2015, S. $987 \mathrm{ff}$. mit w. Nachw. zum Streitstand.

$52 B G H$ v. 11. 12. 2013 - 5 StR 240/13 = HRRS 2014 Nr. 163; m. Anm. Jabn JuS 2014, 371; BGHSt 47, 44, 47; BGHSt 45, 321, 326 mit insoweit krit. Anm. Sinner/Kreuzer StV 2000, 114, 116: Der BGH bleibe mit der Rechtsfolgenlösung „auf halbem Wege stecken“. Ablehnend auch Ambos NStZ 2002, 628, 632; El Ghazi/Zerbes HRRS 2014, 209; vgl. auch - bezogen auf die Rechtslage der Schweiz - Cohen, in: Abo Youssef/Töndury (Hrsg.), Der Schutz polizeilicher Güter, 2011, S. 214; Vetterli forumpoenale 2008, $367,369 \mathrm{f}$.

$53 E G M R$ v. 23.10.2014 - Furcht v. Deutschland - 54648/09, Rn. 47; EGMR v. 5. 2. 2008 - Ramanauskas v. Litauen - 74 420/01, Rn. 60; EGMR v. 9.6. 1998 - Texeira v. Portugal - 44/1997/828/1034, Rn. $39=$ EuGRZ 1999, 660 ff.; vgl. auch Esser, Auf dem Weg zu einem europäischen Strafverfahrensrecht, 2002, S. 171 f.; I. Roxin, in: Festschrift 25 Jahre AG Strafverteidigung des DAV, 2009, S. 1070, 1082.

$54 E G M R$ v. 9.6. 1998 - Texeira v. Portugal - 44/1997/828/1034, Rn. 36 mit Anm. Kempf StV 1999, 128 und Anm. Sommer NStZ 1999, 48; EGMR v. 5.2. 2008 - Ramanauskas v. Litauen - 74420/01, Rn.70; Esser, in: Löwel Rosenberg (LR), StPO, 2002, 26. Aufl.2012, Art. 6 EMRK Rn. 263 ff.; F. Meyer, in: Karpenstein/Mayer (Fn. 37), Art. 6 Rn. 155 ff., vgl. auch I. Roxin, in: Festschrift 25 Jahre AG Strafverteidigung des DAV, 2009, S. 1070, 1084. $55 E G M R$ v. 4.11. 2010 - Bannikova v. Russland - 18757/06, Rn. 54.

56 Vgl. EGMR v. 23.10.2014 - Furcht v. Deutschland - 54648/09, Rn. 68 f. mit Bespr. Hauer NJ 2015, 201; Meyer forumpoenale 2015, 176; Petzsche JR 2015, 88; Sinn/Maly NStZ 2015, 379, 381; vgl. auch bereits Esser (Fn. 53), S. 177 f.; Gaede/Buermeyer HRRS 2008, 279; Kühne, Strafprozessrecht, 9. Aufl. 2015, Rn. 537; C. Roxin JZ 2000, 369; I. Roxin, in: Festschrift

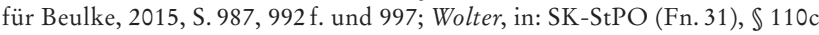
Rn. 9b. 
hen. ${ }^{57}$ Eine solche Engführung kann der Rechtsprechung des $E G M R$ indessen nicht entnommen werden. Das Vorliegen multipler Beweismittel - etwa das Protokoll einer akustischen Überwachungsmaßnahme, ${ }^{58}$ heimliche Gesprächsaufzeichnungen und weitere Zeugen, ${ }^{59}$ TKÜ-Protokolle und forensisches Beweismaterial ${ }^{60}$ - ist in den vom EGMR entschiedenen Fällen die Regel.61 Auch eine (geständige) Einlassung des Provozierten lag meist vor. Und obwohl die nationalen Gerichte bei der Entscheidungsbegründung durchaus zwischen den verschiedenen Beweismitteln differenziert hatten, nimmt der EGMR eine derartige Differenzierung nicht vor. ${ }^{62}$ Bezüglich eines Geständnisses des Provozierten hält der Gerichtshof in „Ramanauskas“ sogar fest, dass "a confession to an offence committed as a result of incitement cannot eradicate either the incitement or its effects." 63

Während der EGMR in den Fällen, in denen es um die heimliche Aushorchung eines Beschuldigten unter Umgehung der Selbstbelastungsfreiheit ging, für die Bewertung des Fairnessverstoßes deutlich auf die intrinsische Beweiskraft der entlockten Informationen (insbesondere der Lockspitzelaussage) und die Verfügbarkeit einer prozessualen Konfrontationsmöglichkeit bzgl. dieser Angaben sowie anderer unterstützender Erkenntnisquellen außerhalb der bemakelten Aussage abgestellt hat, ${ }^{64}$ wird in den Fällen der Tatprovokation mit dem Verdikt des Fairnessverstoßes vorbehaltlos die Verwendung aller Beweismittel verworfen, die in irgendeinem kausalen oder funktionalen Zusammenhang mit der Provokation stehen. ${ }^{65}$ Nichts in diesen Urteilen lässt sich so deuten, dass eine Verurteilung bei Ausschluss der Aussagen der Lockspitzel - und schon gar nicht gestützt auf ein Geständnis - doch fair sein könnte. Auch der Hinweis auf einen alternativ möglichen Straffreistellungsgrund zeigt unzweideutig an, dass nach Auffassung des EGMR am Ende einer durch unzulässige Provokation ausgelösten Strafverfolgung keine Bestrafung stehen darf.

\section{Kriterien für die Grenzziehung zwischen zulässiger und unzulässiger Tatprovokation}

Angesichts der Reichweite des Verwertungsverbots kommt dieses in seiner Wirkung de facto einem Verfahrenshindernis gleich. ${ }^{66}$ Damit stehen und fallen die Möglichkeiten, Ermittlungen unter Verwendung von Tatprovokationen zu betreiben, mit der Frage, wann man es mit einer noch zulässigen

57 Siehe u. IV.2

$58 E G M R$ v. 1.7.2014 - Pareniuc v. Moldawien - 17953/08.

$59 E G M R$ v. 5. 2. 2008 - Ramanauskas v. Litauen - 74 420/01, Rn. 24, 72

$60 E G M R$ v. 4.11. 2010 - Bannikova v. Russland - 18757/06, Rn. 10-12.

61 Schon in der Grundsatzentscheidung "Teixeira“ lagen weitere Beweismittel vor; vgl. EGMR v. 9.6.1998 - Texeira v. Portugal - 44/1997/828/ 1034, Rn. 20.

62 Vgl. EGMR v. 9.6.1998 - Texeira v. Portugal - 44/1997/828/1034, Rn. 32; $E G M R$ v. 26. 10. 2006 - Khudobin v. Russland - 59696/00, Rn. 133; $E G M R$ v. 15. 12. 2005 - Vanyan v. Russland - 53 203/99, Rn. 49. In EGMR v.

4.11.2010 - Bannikova v. Russland - 18757/06, Rn. 15 und in EGMR v. 5.2. 2008 - Ramanauskas v. Litauen - 74420/01, Rn. 72 hatte sich das nationale Gericht entscheidend auf das Geständnis gestützt und daher keine Notwendigkeit für den Ausschluss v. a. der Lockspitzelangaben gesehen.

$63 E G M R$ v. 5.2.2008 - Ramanauskas v. Litauen - 74 420/01, Rn. 72.

$64 E G M R$ v. 10.3.2009-Bykov v. Russland - 4378/02, Rn. 90; EGMR v.

12.5. 2000 - Khan v. Vereinigtes Königreich - 35394/97, Rn. 35 und 37; $E G M R$ v. 5.11.2002 - Allan v. Vereinigtes Königreich - 48539/99, Rn. 43; EGMR v. 12.7.1988 - Schenk v. Schweiz - $10862 / 84$, Rn. 48.

$65 E G M R$ v. 1.7.2014 - Pareniuc v. Moldawien - 17953/08, Rn. 35 EGMR v. 5.2. 2008 - Ramanauskas v. Litauen - 74420/01, Rn. 60; EGMR v. 9.6. 1998 - Texeira v. Portugal - 44/1997/828/1034, Rn. 36.

66 F. Meyer, in: Karpenstein/Mayer (Fn. 37), Art. 6 Rn. 157. oder aber schon unzulässigen Tatprovokation zu tun hat. ${ }^{67}$ Und auch insoweit legt der EGMR Maßstäbe an, die nicht deckungsgleich sind mit denen, die von den deutschen Gerichten entwickelt worden sind. Der EGMR schließt den Einsatz eines agent provocateur zwar nicht schlechthin aus, zieht dem Einsatz von proaktiven Verbrechensbekämpfungsmethoden auf der Grundlage des Fairnessprinzips aber enge Grenzen, in denen sich die deutlich divergierende Einschätzung der rechtsstaatlichen Tolerierbarkeit derartiger Ermittlungsformen niederschlägt. Er stuft Lockspitzeleinsätze als solche als inhärent gefährlich ein, weil er in ihnen eine Tendenz zu manipulativ-anstiftendem Vorgehen erkennt. ${ }^{68}$ Tatprovokationen müssten daher strikten rechtlichen Regeln unterworfen und klar begrenzt werden. Schützende Formen haben die Einhaltung dieser Grenzen sicherzustellen. Der Gerichtshof unterscheidet dabei klar zwischen heimlichen Ermittlungen als solchen und Tatprovokation: „The police may act undercover but not incite". ${ }^{69}$ Der EGMR hat zum Zwecke der Abgrenzung eine dreistufige materielle Zulässigkeitsprüfung entwickelt. ${ }^{70} \mathrm{Er}$ setzt zunächst einmal voraus, dass eine Tatprovokation auf der Basis und im Rahmen einer klaren rechtlichen Grundlage erfolgen muss. ${ }^{71}$ Zum Zweiten muss in Bezug auf die Zielperson im Zeitpunkt der Einwirkung ein plausibler Anlass (good reasons) bestanden haben, sie einer Straftat zu verdächtigen und für geneigt $\mathrm{zu}$ halten, Straftaten zu begehen. ${ }^{72}$ Drittens darf die Einflussnahme der verdeckten Ermittler und V-Personen nicht die Schranke des im Wesentlichen passiven Anreizens zur Tat überschreiten. Hieraus folgt, dass die Tatprovokation bezüglich ihrer Anordnungsvoraussetzungen im nationalen Strafverfahrensrecht hinreichend klar geregelt und im konkreten Fall (versehen mit einer klaren Begründung, die sich zur Tatsachenbasis des Einsatzes verhält und im konkreten Fall Richtlinien für das Verhalten des Lockspitzels aufstellt) autorisiert sein muss. ${ }^{73}$ Des Weiteren müssen Mechanismen zum Missbrauchsschutz existieren und eine effektive Aufsicht bei ihrer Durchführung bestehen. ${ }^{74}$

Eine Grenzüberschreitung liegt jedenfalls dann vor, wenn in Bezug auf die Zielperson im Zeitpunkt der Einwirkung auf diese kein plausibler Anlass (no good reason) bestand, sie einer früheren Straftat $\mathrm{zu}$ verdächtigen und für

67 So auch bereits F. Meyer forumpoenale 2015, 176; Petzsche JR 2015, 88, 90.

68 Vgl. EGMR v. 4.11.2010 - Bannikova v. Russland - 18757/06, Rn. 35; $E G M R$ v. 5. 2. 2008 - Ramanauskas v. Litauen - 74420/01, Rn. 51.

69 So $E G M R$ v. 26. 10.2006 - Khudobin v. Russland - 59696/00, Rn. 28; vgl. ferner EGMR v. 9.6.1998 - Texeira v. Portugal - 44/1997/828/1034, Rn. 36; EGMR v. 4.11.2010 - Bannikova v. Russland - 18757/06, Rn. 35 und Rn. 54. Verdeckte Ermittlungen hält der EGMR eingedenk des gesellschaftlichen Bedürfnisses effektiver Bekämpfung moderner, organisierter Kriminalität in sehr weitem Umfang für zulässig.

70 Zur grundlegenden Entscheidung EGMR v. 9.6.1998 - Texeira v. Portugal - 44/1997/828/1034 vgl. Esser (Fn. 53), S. 173 f. sowie Tyszkiewicz (Fn. 2), S. $106 \mathrm{ff}$.

$71 E G M R$ v. 5.2.2008 - Ramanauskas v. Litauen - 74420/01, Rn. 53; $E G M R$ v. 26.10.2006 - Khudobin v. Russland - 59696/00, Rn.135; eine Präzisierung dieser Anforderungen bleibt der EGMR schuldig; die EMRK ist bei formell-rechtlichen Anforderungen aber in der Regel weniger strikt als das GG.

$72 E G M R$ v. 9.6.1998 - Texeira v. Portugal - 44/1997/828/1034, Rn. 38; $E G M R$ v. 23. 10. 2014 - Furcht v. Deutschland - 54648/09, Rn. 51 m. w. N.; so auch Gaede/Buermeyer HRRS 2008, 279, 284 f.; Kempf StV 1999, 128, 129; Tyszkiewicz (Fn. 2), S. 110.

$73 E G M R$ v. 5.2.2008 - Ramanauskas v. Litauen - 74420/01, Rn.71; EGMR v. 26. 10. 2006 - Khudobin v. Russland - 59696/00, Rn. 135.

$74 E G M R$ v. 5.2.2008 - Ramanauskas v. Litauen - 74420/01, Rn. 53; EGMR v. 26. 10. 2006 - Khudobin v. Russland - 59696/00, Rn. 135, ECHR 2006-XII, und, mutatis mutandis, EGMR v.6.9.1978 - Klass u.a.v. Deutschland - 5029/71, Rn. 52-56, Series A no. 28. 
geneigt zu halten, Straftaten zu begehen. ${ }^{75}$ Jegliche Einwirkung der Behörden auf den Betroffenen hat dann zu unterbleiben. Gleiches muss ab dem Zeitpunkt gelten, in dem eine ursprünglich tatgeneigte Zielperson die Begehung ihr angetragener Straftaten ausdrücklich ablehnt. ${ }^{76}$ Wenn die Zielperson vor dem Erstkontakt ${ }^{77}$ aufgrund hinreichender Anhaltspunkte berechtigterweise als tatverdächtig (in ähnliche kriminelle Aktivitäten verwickelt zu sein oder solche begangen $\mathrm{zu}$ haben) oder tatgeneigt angesehen werden kann, ist eine Einwirkung auf sie dagegen grundsätzlich zulässig. ${ }^{78}$ In Bezug auf den Tatverdacht der Begehung einer ähnlichen Tat wird die Bedeutung des Kriteriums nicht recht klar. Zumindest wird aber deutlich, dass Vorstrafen (criminal record) als solche nicht ausreichen, um eine Tatprovokation zu rechtfertigen. Die Vortaten müssen eine Prädisponiertheit zur Begehung bestimmter Delikte (die Gegenstand der Provokation werden sollen) ausweisen. ${ }^{79}$ Das Fehlen einschlägiger Vorverurteilungen war demgegenüber oftmals Anlass für die Feststellung eines Verstoßes gegen Art 6 EMRK. ${ }^{80}$ Der $E G M R$ verlangt jedoch nicht zwingend, dass der Betroffene bereits zuvor eine Straftat begangen haben muss. Tatverdacht oder gar schlichte Tatgeneigtheit sollen genügen. Für die Annahme einer Tatgeneigtheit hat der Gerichtshof die Vertrautheit der Zielperson mit den Preisen auf dem Drogenmarkt, die Fähigkeit, auf Anfrage hin in kürzester Zeit Drogen liefern zu können, und das finanzielle Interesse an der Durchführung von Transaktionen als Faktoren anerkannt, die auf eine Tatbereitschaft oder eine Involviertheit in kriminelle Aktivitäten hindeuten können. ${ }^{81}$

In beiden Varianten bedarf es jedoch einer soliden Tatsachengrundlage für die Annahme der Polizei, dass der Betroffene bereits erste Schritte zur Begehung der später abzuurteilenden Tat vorgenommen hat. ${ }^{82}$ Gerüchte und Behauptungen sind nicht genug. ${ }^{83} \mathrm{Als}$ sehr problematisch wird eingestuft, wenn die einzige Informationsquelle eine V-Person ist. ${ }^{84}$ Die-

$\overline{75 E G M R}$ v. 9.6.1998 - Texeira v. Portugal - 44/1997/828/1034, Rn. 38; $E G M R$ v. 23. 10. 2014 - Furcht v. Deutschland - 54648/09, Rn. 51 m. w. N.; so auch Gaede/Buermeyer HRRS 2008, 279, 284 f.; Kempf StV 1999, 128, 129; Tyszkiewicz (Fn. 2), S. 110.

76 Verdeckte Ermittler dürfen eine solche Person nicht nochmals kontaktieren, um ihr deliktisches Angebot zu erneuern und auf Deliktsbegehung zu insistieren, EGMR v. 23.10.2014 - Furcht v. Deutschland - 54648/09, Rn. 56, 58.

77 Zur Relevanz dieses Zeitpunktes vgl. EGMR v. 23.10.2014 - Furcht v. Deutschland - 54648/09, Rn. 56.

$78 E G M R$ v. 23.10.2014 - Furcht v. Deutschland - 54648/09, Rn. 50; $E G M R$ v. 4.11.2010 - Bannikova v. Russland - 18757/06, Rn. 38.

$79 E G M R$ v. 4.11.2010 - Bannikova v. Russland - 18757/06, Rn. 41; $E G M R$ v. 29.9.2009 - Constantin u. Stoian v. Rumänien - 23782/06 u. 46629/06, Rn. 55. Dies wurde abgelehnt bei Vorverurteilung eines Drogenabhängigen wegen Drogenbesitzes bezüglich des Handels mit Drogen; noch dazu einer Droge (Heroin), mit der er vorher keine Berührung hatte.

$80 E G M R$ v. 5.2. 2008 - Ramanauskas v. Litauen - 74420/01, Rn. $56 \mathrm{ff}$., 67; $E G M R$ v. 1.7.2008 - Malininas v. Litauen - $10071 / 04$, Rn. 36; EGMR v. 26.10.2006 - Khudobin v. Russland - 59696/00, Rn. 134; EGMR v. 27. 10.2004 - Edwards u. Lewis v. Vereinigtes Königreich - 39647/98 u. $40461 / 98$, Rn. 46.

$81 E G M R$ v. 4.11.2010 - Bannikova v. Russland - 18757/06, Rn. 41 f.; $E G M R$ v. 6.4.2004 - Shannon v. Vereinigtes Königreich - 67537/01; $E G M R$ v. 26.10.2006 - Khudobin v. Russland - 59696/00, Rn. 134; EGMR v. 23. 10. 2014 - Furcht v. Deutschland - 54648/09, Rn. 51 m. w. N.

$82 E G M R$ v. 7.9.2004 - Eurofinacom v. Frankreich - 58 753/00; EGMR v. 6.5.2003 - Sequeira v. Portugal - 73557/01. Unzulässig wäre es, wenn Tatentschluss und Tatplanungen erst nach Hinzutreten des agent provocateur auf dessen Intervention getroffen werden; ausgeschlossen ist aber nicht, dass der Betroffene nach der Infiltration autonom ein deliktisches Angebot macht; vgl. EGMR v. 28. 11. 2000 - Butkevicius v. Litauen - 48297/99.

$83 E G M R$ v. 5.2.2008 - Ramanauskas v. Litauen - 74420/01, Rn.67; $E G M R$ v. 15.12. 2005 - Vanyan v. Russland - 53 203/99, Rn. 49.

$84 E G M R$ v. 26. 10.2006 - Khudobin v. Russland - 59696/00, Rn. 134. se Anhaltspunkte müssen auch bekannt sein, bevor der Erstkontakt stattfindet oder jedenfalls bevor die Einwirkung beginnt. ${ }^{85}$ Davon hängt aus der Sicht des EGMR ganz maßgeblich $\mathrm{ab}$, ob eine laufende kriminelle Unternehmung infiltriert oder eine kriminelle Handlung proaktiv ausgelöst wurde. ${ }^{86}$ Auch wenn sich später herausstellen sollte, dass der Betroffene schon zuvor einschlägige Taten begangen oder erste Schritte unternommen hatte, ist dies für die Beurteilung der Zulässigkeit zum Zeitpunkt des Tätigwerdens irrelevant.

Die Unzulässigkeit des Lockspitzeleinsatzes kann sich schließlich aus dem Umstand ergeben, dass sich dieser nicht darauf beschränkt, im Wesentlichen passiv (essentially passive) $\mathrm{zu}$ ermitteln, sondern er die Zielperson zur Begehung einer Straftat veranlasst, die sonst nicht begangen worden wäre. ${ }^{87}$ Die Frage ist, was das konkret bedeutet. ${ }^{88}$ Der Begriff der passiven Einwirkung kann nicht wörtlich genommen werden, da der EGMR auch das Besorgen von Transportmitteln als noch zulässig eingestuft hat; 89 entscheidend ist, dass von den Lockspitzeln keine Initiative ausging und sie auch keine Gestaltungsherrschaft über die Art der Tatausführung gehabt haben, sondern sich vielmehr in das von der Zielperson beherrschte Geschehen einfügen. ${ }^{90}$ Als zulässigen begleitenden Einstieg in ein laufendes kriminelles Geschehen stuft der EGMR auch Lockangebote bzw. Kontaktaufnahmen unter Vorspiegelung der Absicht zum Geschäftsabschluss ein. ${ }^{91}$ Bei Serientaten ist auch die Simulation einer typischen Tatsituation zulässig. ${ }^{92}$

Umgekehrt hat der EGMR in einer Reihe von Fällen bereits die Initiierung von Testkäufen oder Anreizsituationen als unzulässig gewertet, ${ }^{93}$ ohne die Trennlinie scharf herauszuarbeiten. Dies hat seine wesentliche Ursache darin, dass der EGMR die Korrelation zwischen dem zweiten und dritten Kriterium nicht klar herausstellt. Es scheint offensichtlich, dass die Bewertung der Zulässigkeit einer Einwirkung hinsichtlich ihrer Intensität danach variiert, ob es sich um eine vorbestrafte und (einer ähnlichen Tat) konkret verdächtige Person handelt oder um eine unverdächtigte, objektiv nicht einmal tatgeneigte Person. Die Art der Einwirkung müsste also in einem funktionalen Zusammenhang mit dem Anlass stehen und diesem angemessen sein. Hieraus erklärt sich letztlich die grundsätzliche Unzulässigkeit von Testkäufen, Anreizsituationen oder ähnlichen Initiativen in Bezug auf unverdächtige und nicht tatgeneigte Personen. ${ }^{94}$ Bei bloß

85 So auch eindeutig EGMR v. 1.7.2014 - Pareniuc v. Moldawien 17953/08, Rn. 8; EGMR v. 7.9.2004 - Eurofinacom v. Frankreich $58753 / 00$.

86 Vgl. EGMR v. 4.11.2010 - Bannikova v. Russland - 18757/06, Rn. 43, 47; EGMR v. 1.7. 2008 - Malininas v. Litauen - 10071/04, Rn. 36 f.; EGMR v. 6.5.2003 - Sequeira v. Portugal - 73557/01, ECHR 2003-VI; EGMR v. 7. 9. 2004 - Eurofinacom v. Frankreich - 58753/00.

$87 E G M R$ v. 9.6. 1998 - Texeira v. Portugal - 44/1997/828/1034, Rn. 38 f.; EGMR v. 5. 2. 2008 - Ramanauskas v. Litauen - 74 420/01, Rn. 55; EGMR v 4.11.2010 - Bannikova v. Russland - 18757/06, Rn.37; EGMR v. 23. 10. 2014 - Furcht v. Deutschland - 54648/09, Rn. 48.

88 Vgl. hierzu auch die Analyse der einschlägigen Rechtsprechung des EGMR bei Tyszkiewicz (Fn. 2), S. $111 \mathrm{ff}$.

$89 E G M R$ v. 6.5.2003 - Sequeira v. Portugal - 73557/01, ECHR 2003-VI. 90 Vgl. Tyszkiewicz (Fn. 2), S. 113 f. zum Fall Sequeira; zum Beispiel eines Dauerdelikts EGMR v. 7. 9.2004 - Eurofinacom v. Frankreich - 58753/00. $91 E G M R$ v. 7.9.2004 - Eurofinacom v. Frankreich - 58753/00.

$92 E G M R$ v. 24.6. 2008 - Miliniené v. Litauen - 74355/01, Rn. 37: criminal conduct simulation model.

93 Z. B. $E G M R$ v. 15.12. 2005 - Vanyan v. Russland - 53 203/99, Rn. 49; $E G M R$ v. 26. 10. 2006 - Khudobin v. Russland - 59696/00, Rn. 134: Kontaktaufnahme und Heroinbestellung durch V-Person ohne vorherige Hinweise auf Tatgeneigtheit.

$94 E G M R$ v. 15. 12.2009-Burak Hun v. Türkei - 17570/04, Rn. 44. 
tatgeneigten Personen wird ebenfalls die proaktive Schaffung solcher Situationen unzulässig sein. ${ }^{95}$

Als Fälle nicht mehr passiver und mithin unzulässiger Einwirkung (auch bei verdächtigen oder tatgeneigten Personen) nennt der EGMR: die beharrliche Erneuerung von Angeboten trotz Verweigerung der Zielperson, ${ }^{96}$ das Insistieren mit Preisen, die den Marktwert übersteigen ${ }^{97}$, sowie das Appellieren an das Mitgefühl der Zielperson, beispielsweise durch das Vortäuschen von Entzugserscheinungen ${ }^{98} .99$ Die Unzulässigkeit kann sich des Weiteren daraus ergeben, dass auf die Zielperson Druck (pressure) ausgeübt wird, mit dem Ziel, diese zu veranlassen, das Delikt zu begehen ${ }^{100}$ wobei allerdings das Ausmaß des noch zulässigen Drucks nicht weiter bestimmt wird. Nicht erforderlich ist dabei, dass die direkte Einwirkung durch staatliche Behörden erfolgt. Ein Handeln von Privatpersonen, das mit den Strafverfolgungsbehörden abgestimmt ist oder mit deren Wissen erfolgt, genügt. ${ }^{101}$ Die Einbindung von Privaten als solche ist nicht per se unzulässig. ${ }^{102}$

\section{Die Verbindlichkeit der Vorgaben des EGMR}

Die Entscheidungen des $E G M R$ sind autoritativ für die Interpretation der Konventionsrechte. Sie haben aber keine Gesetzeswirkung, sondern sind im Nationalstaat umzusetzen. Welche Verbindlichkeit ihnen innerstaatlich zukommt, ist durch den innerstaatlichen Rang der EMRK und die mitgliedstaatlichen Grundsätze zu ihrer Implementierung vorgezeichnet. Hiervon hängt nicht nur die Bewertung des jüngsten Kammerbeschlusses des $B \operatorname{VerfG}$ ab, sondern auch, ob die Fortsetzung der bisherigen Praxis der Strafgerichte sich ändern muss, um den Vorgaben des EGMR gerecht zu werden.

\section{Allgemeine Grundsätze}

Die Entscheidungen des EGMR sind für die Parteien des Verfahrens (inter partes) nach Art. 46 Abs. 1 EMRK verbind-

$95 \mathrm{Vgl.EGMR}$ v. 26.10.2006 - Khudobin v. Russland - 59696/00, Rn. 134: kein echter Testkauf, sondern proaktive Suche von V-Person nach jemandem in der Szene, der Heroin verkauft; sehr ähnlich EGMR v. 24. 4. 2007 - V. v. Finland - $40412 / 98$, Rn. 70 f.; EGMR v. 15. 12.2005 Vanyan v. Russland - 53 203/99, Rn. $45-50$.

$96 E G M R$ v. 5.2.2008 - Ramanauskas v. Litauen - 74420/01, Rn.67; EGMR v. 1.7.2014 - Pareniuc v. Moldawien - 17953/08, Rn. 39: blatant prompting and incitement; wobei der $E G M R$ anerkennt, dass eine Verweigerung auch dann gegeben sein kann, nachdem die Zielperson zunächst auf das Ansinnen eingegangen ist, vgl. EGMR v. 23.10.2014 - Furcht v. Deutschland - 54648/09, Rn. 58.

$97 E G M R$ v. 1.7.2008 - Malininas v. Litauen - 10071/04, Rn. 37

$98 E G M R$ v. 15.12.2005 - Vanyan v. Russland - 53203/99, Rn. 11.

$99 E G M R$ v. 4.11.2010 - Bannikova v. Russland - 18757/06, Rn. 47; EGMR v. 23. 10. 2014 - Furcht v. Deutschland - 54648/09, Rn. 52 m. w. N.; vgl. Tyszkiewicz (Fn.2), S. 110, die hieraus den Schluss zieht, dass damit „von vornherein sämtliche Anreiz- und Druck-Situationen aus dem Bereich zulässiger Tatprovokation“ ausscheiden. In der Rechtsprechung des EGMR findet diese These in dieser Absolutheit keine Bestätigung.

$100 E G M R$ v. 6.5.2003 - Sequeira v. Portugal - 73557/01, ECHR 2003VI.

101 Die Behörden treffen Nachforschungspflichten, wenn objektive Anhaltspunkte für eine unzulässige Verleitung durch Private vorliegen, EGMR v. 11.2. 2014 - Sandu v. Moldawien - 16463/08, Rn. 38.

$102 E G M R$ v. 24.6.2008 - Miliniené v. Litauen - 74355/01, Rn. 37 f. Die Grenze des Zulässigen wird im Einklang mit den allgemeinen Grundsätzen überschritten, wenn Polizei Privatpersonen zur aktiven Verleitung auf nicht verdächtige Personen ansetzt, EGMR v. 21.2.2008 - Pyrgiotakis v. Griechenland - 15100/06; anders wenn Provokation durch Private (investigativer Journalist) ohne Wissen der Polizei erfolgte, EGMR v. 6.4.2004Shannon v. Vereinigtes Königreich - 67537/01. lich. ${ }^{103}$ Die Feststellung einer Konventionsverletzung löst eine Umsetzungspflicht aus, die darauf gerichtet ist, die Rechtsverletzung abzustellen und ihren Folgen soweit wie möglich abzuhelfen. ${ }^{104}$ Die Wahl der Mittel zu ihrer Erfüllung obliegt dem verurteilten Mitgliedstaat. ${ }^{105}$ Unter bestimmten Voraussetzungen macht der EGMR jedoch ausnahmsweise spezielle Vorgaben zur Wiedergutmachung der festgestellten Konventionsverletzung. ${ }^{106}$ Der Auswahlspielraum kann sich reduzieren, wenn nur bestimmte Maßnahmen einen andauernden Verstoß effektiv abstellen können; auch systemische Mängel können den EGMR veranlassen, ein bestimmtes Vorgehen einzufordern. ${ }^{107} \mathrm{Im}$ „Furcht“-Urteil limitiert der EGMR den Selektionsspielraum, indem er ein Mittel des nationalen Verfahrensrechts (konkret: die Strafzumessungslösung) disqualifiziert und für die übrigen einen strengen Richtwert setzt (Beweisverwertungsverbot).

Die Verbindlichkeit der Vorgaben des EGMR reicht (erga omnes) über den Einzelfall hinaus, weil dessen Rechtsprechung zur Auslegung des jeweils einschlägigen Menschenrechts bei der Prüfung der EMRK-Konformität nationaler Hoheitsakte stets heranzuziehen und unter den Bedingungen des konkreten Falles zu berücksichtigen ist. ${ }^{108}$ Alle staatlichen Behörden und Gerichte sind infolge ihrer Bindung an Recht und Gesetz (Art. 20 Abs. 3 GG) zur Berücksichtigung der EGMR-Rechtsprechung verpflichtet. ${ }^{109}$ Bestehen Auslegungs- und Abwägungsspielräume, ist den Wertungen des EGMR im Rahmen der geltenden methodischen Standards Vorrang einzuräumen. ${ }^{110}$ Der EGMR-Rechtsprechung wird mithin eine allgemeine normative Orientierungs- und Leitfunktion beigemessen, ${ }^{111}$ die sich sogar zu einer Präzedenzwirkung verdichten kann. ${ }^{112}$

Andererseits hebt das $B \operatorname{Verf} G$ aber auch hervor, dass die „Auswirkungen auf die nationale Rechtsordnung“ bei der konventionsfreundlichen Auslegung Beachtung finden müssen. Die Rechtsprechung des EGMR sei „möglichst schonend in das vorhandene, dogmatisch ausdifferenzierte nationale Rechtssystem einzupassen." 113 Dies gelte insbesondere dann, „wenn es sich um ein in seinen Rechtsfolgen ausbalanciertes Teilsystem des innerstaatlichen Rechts handelt, das verschiedene Grundrechtspositionen miteinander zum Ausgleich bringen will“. ${ }^{114}$ Es sei dann Aufgabe der nationalen

103 Esser StV 2005, 348, 349 ff.; C. Roxin JZ 2000, 369.

$104 E G M R$ v. 26. 02. 2004 - Görgülü v. Deutschland - 74969/01, Rn. 64. 105 Wenzel, in: Karpenstein/Mayer (Fn.37), Art. 41 Rn. 1; EGMR v. 13. 7. 2000 - Scozzari u. Giunta v. Italien - $39221 / 98$ u. 41693/98, Rn. 249. $106 E G M R$ v. 31.10.1995 - Papamichalopoulos u.a.v. Griechenland 14 556/89; EGMR v. 23.3. 2010 - Oyal v. Türkei - 4864/05, Rn. 102; EGMR v. 8. 7. 2004 - Ilascu u. a.v. Moldawien u. Russland - 48 787/99= NJW 2005, 1849, Rn. 490; EGMR (GK) v. 8. 4.2004 - Assanide v. Georgien - 71 503/01 = EuGRZ 2004, 268, Rn. 203; EGMR v. 22.6.2004 - Broniowski v. Polen 31 443/96 = EuGRZ 2004, 472, Rn. 194. Gestützt werden die Anordnungen entweder auf Art. 41 EMRK oder - zumeist - Art. 46 EMRK, Brener, in: Karpenstein/Mayer (Fn. 37), Art. 46 Rn. 6; Çali/Koch HRLR 14 (2014), 301, 309 Fn. 34.

107 Fallgruppen bei Breuer, in: Karpenstein/Mayer (Fn. 37), Art. 46 Rn. $9 \mathrm{ff}$.

108 BVerfGE 111, 307, 324, $329=$ JZ 2004, 1171 mit Anm. E. Klein; Peters/Altwicker, Europäische Menschenrechtskonvention, 2. Aufl.2012, \37 Rn. 24; Esser StV 2005, 348, 352 ff.; C. Roxin JZ 2000, 369; I. Roxin, in: Festschrift 25 Jahre AG Strafverteidigung des DAV, 2009, S. 1070, 1079. $109 B \operatorname{VerfGE} 111,307,329$ f.; 128, 326, 368.

$110 B$ VerfGE 111, 307, 329; 128, 326, 365.

111 Vgl. BVerfGE 128, 326, 368 = JZ 2011, 845 (dazu Streng JZ 2011, 827 und Volkmann JZ 2011, 835); BVerwGE 110, 203, 210, 214.

$112 B \operatorname{VerfGE}$ 128, 326, 368 f.; Mayer, in: Karpenstein/Mayer (Fn. 37), Einl. Rn. 81; Grabenwarter JZ 2010, 857, 861.

113 Vgl. BVerfGE 111,307, 327; 128, 326, 371 .

$114 B \operatorname{VerfG}$ NJW 2004, 3407, 3411; Bergmann, Handlexikon der Europäischen Union, 5. Aufl. 2015, Stichwort: Bindungswirkung von EGMR-Entscheidungen. 
Gerichte, eine Entscheidung des EGMR in den betroffenen Teilrechtsbereich der nationalen Rechtsordnung einzupassen. Lassen das Grundgesetz oder das einfache deutsche Gesetzesrecht eine Umsetzung der materiellen Konventionsvorgaben nicht mehr in methodisch vertretbarer Weise, d.h. im Rahmen der anerkannten Auslegungs- und Anwendungsmethodik, ${ }^{115} \mathrm{zu}$, scheidet diese aus. ${ }^{116}$ Die Berücksichtigung der EGMR-Rechtsprechung dürfe insbesondere nicht dazu führen, dass der Grundrechtsschutz nach dem Grundgesetz eingeschränkt wird. ${ }^{117}$

Infolge des fehlenden Verfassungsrangs der EMRK gelten diese Grundsätze (für die Umsetzung im einfachen Recht) nicht für die Auslegung des Grundgesetzes durch das $B$ VerfG. Zur Frage seiner eigenen Bindung an Urteile des $E G M R$ bei der Verfassungsinterpretation hat das $B$ Verf $G$ dahingehend Stellung bezogen, dass es (beruhend auf der Völkerrechtsfreundlichkeit des Grundgesetzes und seiner inhaltlichen Ausrichtung auf die Menschenrechte) die Wertungen der EMRK bei der Prüfung des einschlägigen Grundrechts (im Rahmen des methodisch Vertretbaren) einbezieht, ${ }^{118}$ - nicht zuletzt um einer Verurteilung Deutschlands durch den $E G M R$ vorzubeugen. ${ }^{119}$

\section{Umsetzung in der jüngsten Kammerrechtsprechung des BVerfG}

Für die Umsetzung der EGMR-Vorgaben zur Tatprovokation waren die Vorzeichen für die bundesdeutsche Praxis mit dem deutlichen Veto des EGMR gegen die Strafzumessungslösung eigentlich klar, die Aufgabe der Strafzumessungslösung schien indiziert. Die Auffassung der 2. Kammer des Zweiten Senats des BVerfG, die Berücksichtigung der unzulässigen Tatprovokation im Rahmen der Strafzumessung genüge den Anforderungen des Rechts auf ein faires Verfahren, ${ }^{120}$ vermag nicht zu überzeugen und zeigt den Strafgerichten bei genauer Analyse auch keinen gangbaren Weg auf, sich künftig von den Fesseln der EMRK zu befreien.

Für den konkreten Fall hebt die Kammer zunächst die Unterschiedlichkeit der Sachverhalte hervor. ${ }^{121}$ Bei veränderter oder anders gelagerter Sach- und Rechtslage wäre ein Abweichen von den Vorgaben des EGMR möglich. ${ }^{122}$ Und der nun zur Entscheidung anstehende Sachverhalt weicht auch tatsächlich in gewissen Aspekten von demjenigen in der Rechtssache Furcht ab. Die Verurteilung stützte sich primär auf Geständnisse der (provozierten) Angeklagten, während auf Aussagen der V-Person zur teilweisen Widerlegung der Einlassungen verzichtet wurde. Im EGMR-Fall waren dagegen neben dem Geständnis auch Vernehmungs-

115 Zur konventionskonformen Auslegung vgl. BVerfGE 74, 370; 111, 307, 323 ff.; BVerfG NJW 2003, 344, 345; BVerfG StV 2008, 1, 2 f.; Woblers/ Gaede NStZ 2004, 9, $12 \mathrm{f}$.

$116 B \operatorname{VerfGK} 11,153,161, B \operatorname{VerfGE} 111,307,315,329 ; 128,326,371$; $B$ VerfG, Beschluss v. 22.10.2014 - 2 BvR 661/12, Rn. 129; Mayer, in: Karpenstein/Mayer (Fn. 37), Einleitung Rn. 88; zur Görgülü-Entscheidung Cremer EuGRZ 2004, 683 ff.; Meyer-Ladewig/Petzold NJW 2005, $15 \mathrm{ff}$.

$117 B \operatorname{VerfGE} 128,326,367$ f.; vgl. $B \operatorname{Verf} G E$ 74, 358, 370; 83, 119, 128; 111, 307, 317; 120, 180, $200 \mathrm{f} .=\mathrm{JZ} 2008,627$ mit Anm. Starck.

$118 B$ VerfGE 111, 307, 322 f.; 128, 326, 365; vgl. auch Sauer ZaöRV 2005, $41,46 \mathrm{ff}$., $51 \mathrm{ff}$.

$119 B \operatorname{Verf} G E$ 128, 326, Rn. 86, 90; als Transmissionsriemen zur Einbeziehung der EMRK-relevanten Aspekte könne dabei das verfassungsimmanente Verhältnismäßigkeitsprinzip dienen, Rn. 94.

$120 B \operatorname{VerfG}$, Beschluss v. 18.12.2014 - 2 BvR 209/14, 240/14, 262/14, Rz. 46.

$121 B$ VerfG, Beschluss v. 18.12.2014 - 2 BvR 209/14, 240/14, 262/14, Rz. 47 ff.; krit. dazu Jäger JA 2015, 473, 475; siehe auch Satzger JURA (JK) 2015, 660 .

$122 B \operatorname{VerfGE}, 111,307,329$. protokolle der verdeckten Ermittler verlesen und (die Einlassung partiell widerlegend) verwertet worden. Derartige Abweichungen sind nun aber aus Sicht des EGMR gar nicht entscheidungserheblich. ${ }^{123}$ Bei genauer Analyse des Fallrechts sind die Vorgaben für den vom $B V \operatorname{Verf} G$ behandelten Sachverhalt eindeutig: Alle mit der Provokation zusammenhängenden Beweismittel sind unverwertbar und selbstverständlich darf auch ein Geständnis nicht verwertet werden. Der Versuch des distinguishing geht somit schon deswegen fehl, weil er auf einer Fehlinterpretation der EGMR-Rechtsprechung aufbaut. Des Weiteren ist in Erinnerung zu rufen, dass das Aufweichen bzw. Ignorieren der vom EGMR ausdrücklich vorgegebenen Limitierung der zulässigen Kompensationsmittel über das Instrument der Gesamtbewertung den nationalen Gerichten auch methodisch gesehen verschlossen ist. Diese Vorgehensweise steht von vornherein nur dem EGMR aufgrund seines besonderen Entscheidungsumfelds und der notwendigen Rücksichtnahme auf die Vielzahl und Verschiedenheiten der Rechtsordnungen im Europarat offen. ${ }^{124}$

Richtigerweise wäre das $B V \operatorname{erf} G$ gehalten gewesen, der Frage nachzugehen, ob sich die Vorgaben des EGMR im Rahmen der anerkannten Auslegungs- und Anwendungsmethodik in das vorhandene nationale Rechtssystem einpassen lassen und mit den Vorgaben des Grundgesetzes vereinbar sind. Das $B$ VerfG lässt insoweit etwas kryptisch verlauten, dass die Rechtsprechung des EGMR und die Strafzumessungslösung des $B G H$ unterschiedliche dogmatische Ansätze verfolgen würden, ohne dies weiter zu vertiefen. ${ }^{125}$ Stattdessen wird darauf verwiesen, dass die Strafzumessungslösung Ausfluss eines ausbalancierten Teilsystems des (Beweis-)Rechts sei, ${ }^{126}$ was man sicherlich schon für sich gesehen mit einem Fragezeichen versehen darf. Aber selbst wenn eine schonende Einfügung nicht ohne Weiteres möglich wäre, wären die Strafgerichte dadurch allein nicht von ihrer Berücksichtigungspflicht befreit. Dies wäre erst dann der Fall, wenn die Umsetzung der EGMR-Vorgaben methodisch unvertretbar wäre. Wenn das $B \operatorname{VerfG}$ konstatiert, dass die Strafgerichte mit ihrer Kombination aus Strafzumessungsund Beweiswürdigungslösung die Rechtsprechung des $E G M R$ schonend in das vorhandene, dogmatisch ausdifferenzierte nationale Rechtssystem eingefügt haben, „ohne dass ihr Vorgehen nach den anerkannten Methoden der Gesetzesauslegung nicht mehr vertretbar erscheint", 127 wird damit der Gradmesser der methodischen Vertretbarkeit verzerrt dargestellt und liegt neben der Sache. Entscheidend ist, ob sich der übertragungsfähige Kerninhalt der EGMR-Vorgaben im Rahmen des methodisch noch Vertretbaren realisieren lässt.

Und das ist auch dann der Fall, wenn der nationale Gesetzgeber und/oder die nationalen Gerichte die damit einhergehenden Konsequenzen aus kriminalpolitischen Gründen ablehnen. Eine Abkehr von den EGMR-Vorgaben ließe sich nur damit begründen, dass die Strafzumessungslösung grundgesetzlich geboten erscheint. Dies behauptet aber auch die Kammer nicht, die selbst davon ausgeht, dass die Strafzumessungslösung nur einfachrechtlich begründet ist und

123 Siehe oben III.

124 F. Meyer, in: Karpenstein/Mayer (Fn. 37), Art. 6 Rn. 225.

$125 B$ VerfG, Beschluss v. 18.12.2014 - 2 BvR 209/14, 240/14, 262/14, Rz. 42.

$126 B$ VerfG, Beschluss v. 18.12.2014 - 2 BvR 209/14, 240/14, 262/14, Rz. 45.

$127 B$ VerfG, Beschluss v. 18.12.2014 - 2 BvR 209/14, 240/14, 262/14, Rz. 49. 
nicht aus der Verfassung folgt, sondern dass von Verfassungs wegen auch eine andere Reaktion auf die Tatprovokation zulässig wäre. ${ }^{128}$

Festzuhalten bleibt: Für die Strafzumessungslösung kann nicht mehr reklamiert werden, sie genüge den Vorgaben des EGMR. ${ }^{129}$ Letztere sind im Rahmen der verfassungsrechtlichen Grenzen umsetzbar und damit für die Strafgerichte und das BVerfG verbindlich. Dass es an der Zeit ist, Abschied von der Strafzumessungslösung zu nehmen, erkennt letztlich auch das $B \operatorname{Verf} G$ an, wenn es beiläufig den Strafgerichten anheimstellt, zukünftig in vergleichbaren Fällen zu erwägen, ein Verwertungsverbot bezüglich der unmittelbar durch die Tatprovokation betroffenen Beweise auszusprechen. ${ }^{130}$

\section{Unzulässige Tatprovokation: quo vadis?}

Die Strafgerichte werden künftig bei unzulässigen Tatprovokationen den Vorgaben des EGMR gemäß Art. 20 Abs. 3 GG Genüge tun und alle provokationsbezogenen Beweismittel als unverwertbar behandeln müssen. Eine unzulässige Tatprovokation löst damit faktisch ein Bestrafungshindernis für die provozierte Tat aus. ${ }^{131}$ Infolgedessen wird sich die Aufmerksamkeit künftig auf die Frage der Zulässigkeit der Provokation verlagern. Ihr wird damit noch größeres Gewicht zukommen. Infolge der absoluten Wirkung konventionsgemäßer Rechtsfolgen werden sich die grundsätzlichen Bewertungsdivergenzen hinsichtlich der Notwendigkeit und Legitimität des Instruments der Tatprovokation nicht mehr mit der Strafzumessungslösung kaschieren lassen, sondern deutlich zum Vorschein kommen. Der EGMR verstellt sich zwar nicht kriminalistischen Notwendigkeiten, doch ist unzweideutig erkennbar, dass er die Zulässigkeitsschwelle wegen seiner Grundskepsis gegenüber Lockspitzeleinsätzen erheblich niedriger ansetzt als der $B G H$ oder das $B V \operatorname{Verf} G$. Auch hier besteht demnach Anpassungsbedarf, wobei der $E G M R$ wohlberaten wäre, größere terminologische und dogmatische Akkuratesse an den Tag zu legen. Allzu oft ist es nämlich diese Ungenauigkeit, die den Mitgliedstaaten eine Umsetzung erschwert oder Umgehungsoptionen erst eröffnet.

Kritik verdient schon, dass die Einstiegsschwelle der "good reasons" nicht präzise festgelegt wird. Der EGMR müsste in Bezug auf die funktionale Bedeutung des Tatverdachts und dessen limitierende Wirkung deutlicher werden: Wofür und warum kann der Tatverdacht eine Rolle spielen? Warum und wann darf man eine nur tatgeneigte Person einem Normkonformitätstest unterziehen? Man erfährt lediglich, dass (zusätzlich) erste Schritte zur Begehung des späte-

$128 B \operatorname{Verf} G$, Beschluss v. 18.12.2014 - 2 BvR 209/14, 240/14, 262/14, Rz. 45.

$129 \mathrm{Sinn} /$ Maly NStZ 2015, 379, $382 \mathrm{f}$., sehen dagegen Spielraum für eine Aufrechterhaltung der Strafzumessungslösung, wenn das Strafmaß auf Null reduziert wird. Umzusetzen sei dies über ein Absehen von Strafe i.S. des $\$ 60$ StGB. Dies ließe aber auf einen Schuldspruch ohne Strafe hinaus, was nicht mit den Vorgaben des EGMR vereinbar wäre; siehe F. Meyer Forumpoenale 2015, 176, 177; wie hier bereits Esser/Gaede/Tsambikakis NStZ 2011, 140, 142; Endriß/Kinzig NStZ 2000, 271, 272; Gaede/Buermeyer HRRS 2008, 279, 285 f.; I. Roxin, in: Festschrift 25 Jahre AG Strafverteidigung des DAV, 2009, S. 1070, 1084.

$130 B \operatorname{VerfG}$, Beschluss v. 18.12.2014 - 2 BvR 209/14, 240/14, 262/14, Rz. 53.

131 So auch Gaede/Buermeyer HRRS 2008, 279, 286; Tyszkiewicz (Fn. 2), S. 223; Wolter, in: Festgabe BGH, Bd. IV, 2000, S. 980; vgl. auch Beulke, in: SSW-StGB (Fn. 38), Einl. Rn. 110a; sowie Schumann JZ 1986, 66. ren Delikts vorgenommen worden sein müssen, 132 ohne dass klar wird, was das bedeutet. Insgesamt differenziert der $E G M R$ zu wenig nach den verschiedenen Erscheinungsformen der Tatprovokation. Insbesondere die Korrelation zwischen Anlass und zulässigem Maß der Einwirkung wird nicht herausgearbeitet. Insofern wird zwar die Passivitätsschwelle für die Einwirkung im Vergleich relativ niedrig angesetzt. Eine differenzierungsmächtige Taxonomie lässt sich den Urteilen aber nicht entnehmen; alles verschwimmt am Ende in einer Gesamtbewertung.

Ohne an dieser Stelle selbst eine umfassende Klassifizierung vornehmen zu können, soll als Ausblick dennoch eine Grobskizzierung gewagt werden: Als legitime Anwendungssituationen einer Tatprovokation erscheinen nur solche, in denen objektiv belegbar ist, dass eine tatgeneigte Zielperson bereits erste Schritte zur Begehung des späteren Delikts getan hat. ${ }^{133}$ Bei anderen tatgeneigten Personen ist allenfalls eine präventive Neigungserforschung denkbar, wofür dann aber die Mittel der StPO nicht zur Verfügung stehen. Solange jedoch kein Anfangsverdacht im strafprozessualen Sinn, sondern lediglich die Erwartung einer zukünftigen Tat vorliegt, kann die Annäherung an die Zielperson bzw. die Infiltration einer kriminellen Struktur nur dann zulässig sein, wenn die Zielperson im Zusammenhang mit einem bereits vorliegenden (provokationstat-externen) Verdacht bezüglich anderer Straftaten steht und ein kriminalistisch-funktionaler Zusammenhang zwischen diesen und der Tat besteht, zu der verleitet werden soll; vor allem bei Serientaten oder phänotypisch ähnlichen Taten.134 Ohne konkreten Tatverdacht oder bei qualitativ anderem Verdacht wäre eine Tatprovokation dagegen per se unzulässig. ${ }^{135}$ Je nach Verdachtsgrad und Deliktsart wäre dann des Weiteren zu differenzieren, wie intensiv die Verleitung der Zielperson ausfallen darf. Scheinkäufe oder kontrollierte Lieferungen, wie sie viele internationale Übereinkommen vorsehen, können dabei als Instrument, das in laufende Ermittlungen eingebettet ist, grundsätzlich zulässig sein, wenn Sie nicht mit weiteren Zwangsoder Verleitungsmomenten (Drohungen, Appell an Empathie, atypisch hoher Erlös) verbunden werden. Ähnliches lässt sich bei Serientaten wohl auch für die Replizierung vermuteter früherer Taten sagen. Die gesammelten Beweismittel könnten dann zur Verurteilung wegen der provozierten Tat ${ }^{136}$ oder zur Verurteilung wegen früherer Taten oder Dauerdelikten, wegen derer rechtmäßig ermittelt wurde, herangezogen werden. ${ }^{137}$

Schließlich muss man sich fragen, ob es angesichts des tiefen Eingriffs in das allgemeine Persönlichkeitsrecht, den die gezielte Einwirkung auf Willensbildung und Tatentschluss bewirkt, ${ }^{138}$ nicht einer speziellen Ermächtigungsgrundlage bedarf, ${ }^{139}$ die sich dezidiert zu den zulässigen Fall-

$132 E G M R$ v. 24. 4. 2014 - Lagutin u.a.v. Russland - 19678/07, 52 340/08, $6228 / 09$.

$133 E G M R$ v. 24. 4. 2014 - Lagutin u. a.v. Russland - 19678/07, 52 340/08, $6228 / 09$.

134 Bei Dauerdelikten könnte sich der Tatverdacht sogar auf dieselbe laufende Tat beziehen.

135 Vgl. auch schon Fischer/Maul NStZ 1992, 7, 11.

136 Vorausgesetzt, es besteht ein kriminalistisch-funktionaler Zusammenhang mit dem Anlass der Tatverleitung.

$137 E G M R$ v. 7.9.2004 - Eurofinacom v. Frankreich - 58753/00; anders Tyszkiewicz (Fn.2), die bei der Zulässigkeit zwischen der Aburteilung wegen provozierter Tat (S. $129 \mathrm{ff}$.) und wegen anderer Taten (S. 146ff.) differenziert.

138 Dazu v. Danwitz (Fn. 10), S. 85 ff.; Fischer/Maul NStZ 1992, 7, 11.

139 Tyszkiewicz (Fn. 2), S. 234 f.; ähnlich schon Maul/Fischer NStZ 1992, $7,13$. 
gruppen und der kriminalistischen Notwendigkeit des Einsatzes verhalten müsste. Gerhard Fezer hätte dies ganz gewiss so gesehen.

\section{Epilog}

Der 2. Strafsenat des $B G H$ hat in einem Urteil v. 10. 6. 2015 (Az. 2 StR 97/14) nunmehr die gebotene Kehrtwende vollzogen und erstmals ein Strafverfahrenshindernis wegen rechtsstaatswidriger Tatprovokation anerkannt. Die schriftlichen Entscheidungsgründe lagen bei Drucklegung dieses Beitrags noch nicht vor. Die Pressemitteilung Nr.91/2015 vom 10.6. 2015 lässt erkennen, dass der Senat trotz massiver Einflussnahme auf die späteren Beschuldigten keinen der vom $B \operatorname{VerfG}$ abstrakt für möglich gehaltenen Ausnahmefälle annehmen konnte, was ein Verfahrenshindernis bereits auf der Grundlage der bisherigen Rechtsprechung begründet und zugleich den offenen Konflikt mit dem EGMR vermieden hätte. Ebenso wenig wollte der Senat offenbar der „Differenzierungslösung“ der 2. Kammer des Zweiten BVerfGSenats folgen und die Anwendung der Furcht-Rechtsprechung auf den konkreten Sachverhalt leugnen. ${ }^{140}$ Die oben dargelegte Reichweite der Bindungswirkung scheint dem $B G H$-Senat klar gewesen zu sein. In Ermangelung der

140 Auch hier beruhten die Feststellungen des $L G$ entscheidend auf den Einlassungen der Angeklagten. schriftlichen Urteilsgründe bleibt für den Moment aber offen, wie der Senat den materiellen Kerngehalt der EGMRRechtsprechung bestimmt, auf den sich die Berücksichtigungspflicht bezieht. Dieser Aspekt ist nicht unwichtig, weil der 2. Senat ein Hintertürchen offen lässt und in den Raum stellt, ob jede rechtsstaatswidrige Tatprovokation die absolute Rechtsfolge eines endgültigen Verfahrenshindernisses nach sich ziehen muss, oder „ob eine abgestufte Lösung je nach der konkreten Schwere der Menschenrechtsverletzung möglich wäre". ${ }^{141}$ Die Antwort scheint aus EGMR-Sicht klar: Eine unzulässige Tatprovokation wiegt intrinsisch so schwer, dass eine andere Rechtsfolge als der Verfahrensabbruch nicht in Betracht kommen kann. Es wäre schön gewesen, wenn die höchsten deutschen Gerichte diese Folgerungen direkt aus unserer eigenen Verfassung geschöpft hätten. ${ }^{142}$

141 Insofern hätte eine Anrufung des Großen Senats wegen grundsätzlicher Bedeutung doch nahe gelegen; sowohl bezüglich der Reichweite der Bindungswirkung als auch insbesondere bezüglich der Rechtsfolge und etwaiger alternativer Lösungswege wäre ein klärender Beschluss zur Sicherung einer einheitlichen Rechtsprechung mehr als förderlich gewesen. Schon dass ein Fall der Innendivergenz deshalb nicht vorgelegen haben soll, weil sich die Rechtslage geändert hätte, überzeugt nur sehr bedingt, denn der verbindliche materielle Kern der Konventionsrechtslage hat sich mit dem „Furcht-Urteil“ überhaupt nicht verändert, sondern allenfalls die Einsicht beim 2. Senat, sich in das Unvermeidliche fügen zu müssen.

142 Zur Ableitung zumindest eines Beweisverwertungsverbots aus der Verletzung des allgemeinen Persönlichkeitsrechts bei Provokation allgemein "Tatgeneigter" ohne Anfangsverdacht schon (bzw. noch) Maul/Fischer NStZ 1992, 7, 13.

\section{Glückwunsch}

\section{Joachim Rückert 70 Jahre}

Das Freiheitsproblem als Kernproblem der Privatrechtsgeschichte. - Im umfangreichen Werk von Joachim Rückert stößt man immer wieder auf diesen und ähnliche Sätze. In den gedrängten Worten bündeln sich mehrere Fäden, an denen der Frankfurter Rechtshistoriker seit langem spinnt. Wenige Andeutungen zeigen die Weite des Horizonts. Freiheit ist für Rückert das wesentliche Prinzip des Privatrechts schlechthin. Gern grenzt er ein freies von einem gebundenen Privatrecht ab. Den Vorzeichenwechsel in der Sattelzeit um 1800 hat er stets im Blick. Damit ist Freiheit nicht nur eine politischwohlfeile Sonntagsfloskel, sondern geltendes Recht. Historisch-kritisch lässt sich bestimmen, ob und inwieweit der moderne Staat bereit ist, den gesetzlich verankerten Grundsatz durchzusetzen. Unklare Grenzen zu einem diffus sozialen Bereich können dabei die juristische Klarheit trüben. Der Rechtshistoriker Rückert, der die Entstehung und Schlagkraft prinzipieller Rechtssätze überblickt, erhebt an dieser Stelle lautstark seine Stimme und zeigt selbstbewusst, welches Gewicht rechtshistorisches Wissen besitzen kann, wenn nur jemand bereit ist, es zur Kenntnis zu nehmen. Die Freiheit, die Rückert im Privatrecht findet und auf den Punkt bringt, ist für ihn mehr als ein Grundrecht oder ein anderes Wort für Privatautonomie. Vielmehr verbinden sich hier die philosophische Tradition, rechtshistorische Linien und ein dogmatisches Auslegungsgebot. Wie kaum ein anderer legt Rückert großen Wert darauf, dass die kantische Lehre vom freiheitlichen Recht alles andere als formal oder leblos daherkommt. Das Gesetz, das Kant als Regel für das menschliche Miteinander formuliert, soll eben gerade ein allgemeines Gesetz der Freiheit sein.

In der Rückbesinnung auf solche Einsichten geht es Rückert immer um ein großes Thema. Er hat zu didaktischen Zwecken die Rechtsgeschichte gern in Zeit- und Sachtypen eingeteilt und findet im Prinzip Freiheit oder im Ausmaß staatlicher Gewalt Wegweiser, um Ordnung in den Stoff zu bringen. Denn getreu der Maxime Leopold von Rankes betont er das Sammeln, Sichten, Ordnen und Werten. Dann ist es möglich, große Erzählungen zu verfassen und sich nicht von der Masse der Einzelheiten überwältigen zu lassen. Mit großer Beharrlichkeit, in den vergangenen Jahren mit dem spürbaren Bedürfnis, den eigenen Erkenntnissen bleibende Beachtung zu sichern, appelliert Rückert in seinen Vorträgen und Aufsätzen daran, die Einsichten der rechtshistorischen Forschung ernstzunehmen. Daraus erwächst kaum verwunderlich große Skepsis gegenüber prinzipienloser Schutzgesetzgebung zugunsten von Verbrauchern oder anderen angeblichen schwachen gesellschaftlichen Gruppen ebenso wie gegenüber einer Zivilrechtswissenschaft, die sich mit dem bloßen Nachvollzug von Gerichtsentscheidungen begnügt. Den Stoff zu durchdringen, um ihn zu beherrschen, bildet dabei eine unausgesprochene Leitlinie. Rückert knüpft bewusst an die Historische Rechtsschule an, die er umfassend erforscht hat. Er ist und war nie ein Neopandektist, wie man andere etikettiert hat, die aus der römisch-rechtlichen Tradi- 